\title{
Diferencias entre el error estándar y desviación estándar de la disposición a pagar: aplicación del método bootstrap en la valoración contingente de un bien o servicio ambiental
}

\author{
Differences between standard error and standard deviation of the willingness to pay: application \\ of the bootstrap method in contingent valuation of an environmental good or service
}

${ }^{1}$ Luis M. Ledesma Goyzueta y ${ }^{2}$ Jorge A. Alarcón Novoa

Resumen

En el presente artículo se muestra, de manera práctica, la inclusión del método bootstrap (Efron, 1979) dentro del proceso de estimación de la disposición a pagar (DAP) de un determinado bien y/o servicio ambiental, bajo el enfoque de la valoración contingente. Considerando los aportes de McLeod y Bergland (1989) y Cooper (1994), se procedió a estimar el error estándar y el intervalo de confianza bootstrap de la DAP, para lo cual se utilizó los datos de un estudio realizado en Ica, Perú (MINAM, 2013), con fines ilustrativos. El propósito principal del documento es exponer las diferencias conceptuales y de cálculo entre el error estándar y la desviación estándar de la DAP, a fin de evitar errores de interpretación de estos estadísticos dentro de un estudio de valoración contingente. Los resultados han mostrado diferencias significativas entre el error estándar y la desviación estándar; asimismo se concluye que la desviación estándar sobrevalora el nivel de variabilidad de la DAP estimada.

Palabras Claves: disposición a pagar, bootstrap, valoración contingente, modelo logit, error estándar.

Clasificación JEL: C15, C35, Q59

\begin{abstract}
In this article is shown, in a practical way, inclusion of the bootstrap method (Efron, 1979) in the process of estimating the willingness to pay (WTP), for a determined environmental service, using the binary choice model for contingent valuation. Considering the contributions of McLeod and Bergland (1989) and Cooper (1994), it is proceeded to estimate the standard error and the confidence interval of the WTP by the bootstrap method, using -for this purpose- the data of a contingent valuation study developed in Peru (MINAM, 2013). Important objective of the paper is to present the conceptual and calculation differences between the standard error and standard deviation of the WTP, in order to avoid misinterpretations of these statistics in a contingent valuation study. Results showed significant differences between error and standard deviation, also it was concluded that the standard deviation overvalue the variability of the estimated WTP.
\end{abstract}

Keywords: willingness to pay, bootstrap, contingent valuation, logit model, standard error.

JEL Classification: C15, C35, Q59

\section{Introducción}

Los métodos de valoración contingente tienen como objetivo estimar el valor económico de un bien o servicio basándose en el cambio del nivel de bienestar que experimentan los agentes cuando se presenta un cambio en la dotación de dicho bien o servicio. Este método es comúnmente aplicado para valorar bienes o servicios ambientales, debido a que no presentan un mercado definido o precio de transacción.
El modelo tipo referéndum desarrollado por Hanemann (1984), basado en el enfoque de diferencias de la función indirecta de utilidad, es uno de los criterios más utilizados dentro de la valoración contingente. Dentro de ese enfoque, destaca el uso de modelos de estimación con variables de respuesta binaria, tales como el modelo LOGIT o PROBIT, los cuales son aplicados para estimar la disposición a pagar (DAP), con el objetivo ulterior de aproximar el valor económico de un determinado bien o servicio ${ }^{3}$.

\footnotetext{
${ }^{1}$ Facultad de Ciencias Matemáticas, Universidad Nacional Mayor de San Marcos, Lima, Perú. E-mail: luis.ledesma@unmsm.edu.pe. ${ }^{2}$ Facultad de Economía y Planificación, Universidad Nacional Agraria La Molina, Lima, Perú. E-mail: jalarcon@lamolina.edu.pe.

${ }^{3}$ A partir del trabajo de Bishop y Herberlein (1979), se incorporó un formato de pregunta binaria o dicotómica en las encuestas de valoración contingente, donde los individuos deciden si adquieren un detemindo bien, a travéz de un pago o desembolso, o simplemente se niegan a dicha "compra".
} 
Yoo (2011) hace mención que la distribución muestral de la DAP para bienes ambientales, convencionalmente es obtenida mediante procedimientos de simulación, en donde destacan la aproximación de Cameron (Cameron, 1991), el método bootstrap (Efron, 1979), el método Jackniffe (Mcleod y Bergland, 1989) y el método de simulación Montecarlo desarrollado por Krinsky y Robb (1986). En ese sentido, con la aplicación de dichas técnicas es posible estimar el error estándar y/o el intervalo de confianza de la DAP. ${ }^{4}$

En los últimos años, se han venido desarrollando estudios que comparan el desempeño de distintos métodos para estimar los intervalos de confianza de la DAP, destacándose los trabajos de Hole (2007), Bliemer y Rose (2013) y Gatta y Marcucci (2014). Por otro lado, Carson y Czajkowski (2013) han demostrado que, bajo ciertas condiciones, los cálculos del error estándar e intervalo de confianza de la DAP presentan algunos problemas cuando son estimados mediante modelos de elección discreta, proponen como solución alternativa una reparametrización de la variable costo del modelo mediante una transformación exponencial.

Respecto a los estudios realizados en el Perú, no es común observar el detalle metodológico del cálculo del error estándar de la DAP, o incluso del intervalo de confianza, dentro de los resultados de los modelos estimados 5 ; siendo una práctica más común presentar la desviación estándar dentro de las estadísticas descriptivas de la DAP. Es importante tener en cuenta, además, que existen paquetes estadísticos que incorporan distintos métodos de estimación para obtener el error estándar y los intervalos de confianza de la DAP de manera directa y sencilla. ${ }^{6}$ El objetivo del presente artículo es mostrar en detalle el procedimiento del cálculo del error estándar y el intervalo de confianza de la DAP dentro de un estudio de valoración contingente de formato binario. Asimismo, comparar el valor estimado del error estándar con la desviación estándar de la DAP, dando pie a describir las diferencias que presentan dichos estadísticos. La hipótesis central es que el error estándar difiere significativamente de la desviación estándar, resultando ser un mejor estimador de la variabilidad de estimación de la DAP, en el contexto de Valoración Contingente.

Para fines ilustrativos, en este trabajo se han utilizado datos del estudio "Diseño e Implementación de un Esquema de Retribución por Servicios Ecosistémicos ${ }^{4}$ Cooper (1994) comparó el desempeño de los métodos de simulación señalados, encontrando que no existe un método superior entre ellos. Por lo tanto, Yoo (2011) menciona que la elección de un método para la construcción de intervalos de confianza para la DAP debe ser realizada de manera ad hoc.

${ }^{5}$ Por ejemplo, revisando los trabajos de Loyola (2007) y Loyola y Soncco (2007), los cuales fueron los únicos estudios de valoración contingente realizados en el Perú dentro de la lista de estudios referentes del proyecto de la Guía de Valoración Económica de Impactos Ambientales (publicado mediante Resolución Ministerial N 387-2013-MINAM), se identificó la no inclusión del detalle metodológico del cálculo del error estándar e intervalo de confianza de la DAP en el contenido de los estudios.

${ }^{6}$ Entre otros, el software STATA presenta un comando denominado "wtpcikr", el cual permite obtener los intervalos de confianza de la DAP basado en el método propuesto de Krinsky y Robb (1986).
Hidrológicos en la Cuenca del Río Cañete", realizado por el Ministerio del Ambiente del Perú (MINAM) en el 2013, en el cual se estimó la DAP de los consumidores de agua potable, de la localidad de Cañete, para evaluar el nivel de aporte que tendrían para establecer un fondo de conservación de la parte alta de la cuenca del rio Cañete. Considerando esta información, se procedió a estimar el error estándar y el intervalo de confianza de la $\mathrm{DAP}^{7}$, a través de la obtención de la distribución empírica de la DAP, mediante la aplicación del método bootstrap. Es preciso señalar que dichos estadísticos no fueron calculados y/o presentados en el estudio original.

Si bien existen técnicas para estimar el error estándar o el intervalo de confianza de la DAP, estos no son calculados o detallados en algunos estudios realizados en el Perú, es por ello que en el presente artículo se expone de manera práctica la obtención del error estándar de la DAP a partir de un estudio ya realizado. Además de esta introducción, este artículo está constituido por tres secciones más: una sección metodológica, luego la presentación de resultados, y finalmente las conclusiones de rigor.

\section{Metodología}

La presente sección expone el método y los datos utilizados en la estimación del error estándar de la DAP, en un contexto de aplicación del método de valoración contingente de formato binario. En ese sentido, se utilizó como fuente de referencia la base de datos del estudio del MINAM (2013) con el objetivo de obtener la distribución empírica de la DAP, a través de la inclusión del método bootstrap en el modelo.

Posteriormente, a partir de las estimaciones obtenidas, se discuten las diferencias conceptuales y de interpretación que presenta el error estándar de la DAP, con respecto a la desviación estándar. En el Anexo se muestran los códigos programados (en lenguaje R) ${ }^{8}$ de los procedimientos de estimación.

\subsection{Base de datos}

En este estudio se ha tomado como ejemplo ilustrativo la información del estudio desarrollado por el MINAM (2013), específicamente, de la base de datos de encuestas realizadas en la región de Ica, a 470 usuarios domésticos de agua potable, donde el fin fue conocer la disposición de las personas a contribuir en un fondo para la conservación de la parte alta de la cuenca del rio Cañete. De acuerdo a los resultados expuestos en MINAM (2013), el valor estimado de la DAP alcanza los 5,05 soles por mes para los usuarios domésticos de agua potable.

Para fines de comparación, es preciso señalar que la especificación del modelo logit estimado fue la misma que se determinó en el documento del MINAM (2013).

${ }^{8} \mathrm{R}$ Core Team (2015). R: A language and environment for statistical computing. R Foundation for Statistical Computing, Vienna, Austria. URL: <https://www.r-project.org/>. 


\subsection{Cálculo de la disposición a pagar}

Para obtener la fórmula de la DAP, se siguió el planteamiento propuesto por Hanemann (1984), considerando el supuesto de linealidad de una función de utilidad indirecta. Por lo tanto, si se asume que la función de utilidad indirecta tiene relación de dependencia con el nivel de ingresos ( $y$ ) la calidad ambiental $(q)$, así como con otras variables observables $z_{1}, z_{2}, \ldots, z_{m}$, entonces tenemos que:

$$
u_{j}=v_{j}\left(y, z_{1}, z_{2}, \ldots, z_{m} ; q_{j}\right)+\varepsilon_{j}
$$

Donde $j=0$ representa la situación inicial, $j=1$, la situación de cambio (por ejemplo, la mejora en la calidad ambiental) y $\varepsilon_{j}$ representa el componente estocástico ${ }^{9}$. Si para elegir la situación de mejora ambiental un individuo tiene que pagar $A$, las funciones de utilidad indirecta se pueden expresar de la siguiente manera, según situación:

$$
\begin{gathered}
u_{0}=v_{0}\left(y, z_{1}, \ldots, z_{m} ; q_{0}\right)+\varepsilon_{0} \\
u_{1}=v_{1}\left(y-A, z_{1}, \ldots, z_{m} ; q_{1}\right)+\varepsilon_{1}
\end{gathered}
$$

En el caso que $u_{1}>u_{0}$, se presentan incentivos (en términos de bienestar) para aceptar el pago de $A \mathrm{y}$, por ende, conseguir la situación de mejora ambiental $q_{1}$. Si se define $\Delta v$ como el diferencial de utilidad indirecta entre la situación inicial y la situación de mejora ambiental, calculado como $\Delta v=v_{1}-v_{0}$, entonces la condición donde el pago de $A$ es preferible, se puede expresar también como:

$$
\begin{gathered}
u_{1}>u_{0} \\
v_{1}\left(y-A, z_{1}, \ldots, z_{\mathrm{m}} ; q_{1}\right)+\varepsilon_{1}>v_{0}\left(y, z_{1}, \ldots, z_{\mathrm{m}} ; q_{0}\right)+\varepsilon_{0} \\
v_{1}\left(y-A, z_{1}, \ldots, z_{m} ; q_{1}\right)-v_{0}\left(y, z_{1}, \ldots, z_{m} ; q_{0}\right)>\varepsilon_{0}-\varepsilon_{1} \\
\Delta v>\varepsilon_{0}-\varepsilon_{1}
\end{gathered}
$$

Si se considera que las funciones de utilidad indirecta $v_{j}$ son lineales, entonces el $\Delta v$ se puede expresar como:

$$
\begin{gathered}
\Delta v=\left(\alpha_{1}+\beta(y-A)+\sum_{k=1}^{m} \gamma_{1 k} z_{k}\right)-\left(\alpha_{0}+\beta y+\sum_{k=1}^{m} \gamma_{0 k} z_{k}\right) \\
\Delta v=\left(\alpha_{1}+\alpha_{0}\right)-\beta A+\sum_{k=1}^{m}\left(\gamma_{1 k}-\gamma_{0 k}\right) Z_{i} \\
\Delta v=\alpha-\beta A+\sum_{k=1}^{m} \delta_{k} z_{k}
\end{gathered}
$$

Donde $\alpha=\alpha_{1}-\alpha_{0}$ y $\delta_{k}=\gamma_{1 k}-\gamma_{0 k}, \forall k=1, \ldots, m$.

Retomando la expresión (2), se puede definir la probabilidad de aceptar el pago $A$ mediante la expresión $p=P(\varepsilon \leq \Delta v)$, donde $\varepsilon=\varepsilon_{0}-\varepsilon_{1}$. Asimismo, si consideramos que la probabilidad $p$ presenta una forma funcional logística, tenemos que:

$$
p_{i}=\frac{1}{1+\exp (-\Delta v)}=\frac{1}{1+\exp \left\{-\left(\alpha-\beta A_{i}+\delta_{1} z_{1 i}+\ldots+\delta_{m} z_{m i}\right)\right\}}
$$

Reordenando la ecuación (4), obtenemos la especificación LOGIT siguiente:

$\ln \left\{p_{i} /\left(1-p_{i}\right)\right\}=\alpha-\beta A_{\mathrm{i}}+\delta_{1} z_{1 i}+\delta_{2} z_{2 i}+\ldots+\delta_{m} z_{m i}$

En consecuencia, teniendo en cuenta la especificación de la ecuación (5), la estimación de los parámetros $\alpha, \beta$, $\delta_{1}, \ldots, \delta_{m}$ se puede realizar mediante el método de máxima verosimilitud a partir de una especificación LOGIT.

Por otro lado, en el caso que $A$ sea igual a la verdadera valoración que un individuo le asigna a un bien, se presentaría un nivel de indiferencia entre pagar y no pagar dicho monto. Si tenemos que la valoración de un individuo es $C$, entonces se presenta la siguiente situación:

$$
\begin{gathered}
v_{1}\left(y-C, z_{1}, \ldots, z_{m} ; q_{1}\right)+\varepsilon_{1}=v_{0}\left(y, z_{1}, \ldots, z_{m} ; q_{0}\right)+\varepsilon_{0} \\
v_{1}\left(y-C, z_{1}, \ldots, z_{m} ; q_{1}\right)-v_{0}\left(y, z_{1}, \ldots, z_{m} ; q_{0}\right)=\varepsilon_{0}-\varepsilon_{1} \\
\Delta v=\varepsilon \\
\alpha-\beta C+\sum_{k=1}^{m} \delta_{k} z_{k}=\varepsilon
\end{gathered}
$$

Despejando, se obtiene la expresión de la disposición a pagar por cada individuo :

$C_{i}=\left(\alpha+\delta_{1} z_{1 i}+\delta_{2} z_{2 i}+\ldots+\delta_{m} z_{m i}-\varepsilon_{i}\right) / \beta$

Donde $i=1, \ldots, n$, siendo $n$ el tamaño de muestra.

Retomando la ecuación (6), el valor esperado de la disposición a pagar (DAP), considerando los parámetros estimados del modelo LOGIT, tendría la siguiente expresión:

$D A P=E[C]=\frac{\hat{\alpha}+\hat{\delta}_{1} E\left[z_{1 i}\right]+\hat{\delta}_{2} E\left[z_{2 i}\right]+\ldots+\hat{\delta}_{m} E\left[z_{m i}\right]}{\hat{\beta}}$

\footnotetext{
${ }^{9}$ Un supuesto importante detrás del método de valoración contingente consiste en que las funciones de utilidad tienen componentes que son a priori desconocidos, lo cual involucra que la estructura del modelo sea estocástica, es decir, la especificación de las funciones de utilidad incorpora un componente estocástico (Vásquez et al, 2007).
} 


\subsection{Aplicación del método bootstrap en el modelo}

El bootstrap es un método computacional propuesto por Bradley Efron en 1979, que consiste en estimar medidas de precisión de estimadores estadísticos. Una muestra bootstrap $X^{*}=\left(x_{1}{ }^{*}, x_{2}{ }^{*}, \ldots, x_{\mathrm{n}}{ }^{*}\right)$ es obtenida mediante un remuestreo aleatorio con reemplazo, a partir de los elementos de la muestra original, $B$ veces, por lo tanto, se podrá estimar estadísticos para cada una de las muestras bootstrap.
Al respecto, si se remuestrea con reemplazo $B$ veces la muestra original de una encuesta de valoración contingente, el cálculo de la DAP bootstrap se obtiene mediante la ecuación $\sum_{i=1}^{B} E\left[C_{(i)}^{*}\right] / B$, siendo $E\left[C_{(i)}^{*}\right]$ el valor esperado de la disposición a pagar, por cada muestra bootstrap, donde $i=1,2, \ldots, B$, tal como se muestra en la Figura 1.

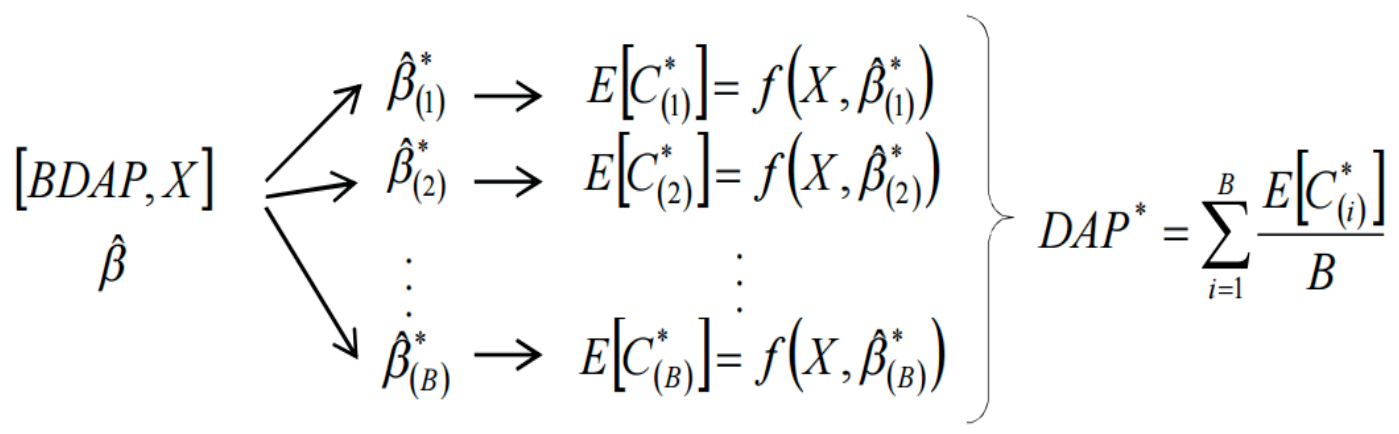

Figura 1. Estimación de la DAP mediante bootstrap

Como se observa en la ecuación (7), cuando se asume una función de utilidad indirecta lineal, la fórmula de la DAP presenta una especificación no lineal en términos de los coeficientes LOGIT, por lo que el cálculo de la varianza de la DAP no se puede obtener de manera directa. Sin embargo, al aplicarse el método bootstrap el cálculo del error estándar de cualquier estadístico es obtenido de manera directa.

En ese sentido, siguiendo el planteamiento de Efron (1979), para obtener el error estándar bootstrap de la DAP, se calcula la desviación estándar del valor esperado $E\left[C_{(i)}^{*}\right]$, donde $i=1,2, \ldots, B$, como se muestra en la siguiente fórmula:

$$
s e_{D A P}^{*}=\sqrt{\frac{\sum_{i=1}^{B}\left(\mathrm{E}\left[C_{i}^{*}\right]-D A P^{*}\right)^{2}}{B-1}}
$$

Por lo tanto, el error estándar calculado mediante bootstrap refleja el nivel de variabilidad del valor esperado de la DAP

\subsection{Diferencias entre la desviación y error estándar de la DAP}

Si se tiene una muestra $x_{1}, x_{2}, x_{3}, \ldots, x_{n}$, el cálculo de la desviación estándar ${ }^{10}$ de las $n$ observaciones de la

\footnotetext{
${ }^{10}$ La desviación estándar es definida como la raíz cuadrada de la varianz de una variable. La varianza es una medida de dispersión que expresa el valor esperado al cuadrado de la diferencia o desviación de una variable respecto a su media, es decir, $E\left[\left(X-\mu_{X}\right)^{2}\right]$, donde $X$ es una variable aleatoria y $\mu_{X}$ es la media de $X$.
}

variable, se obtiene mediante la expresión $\sqrt{\frac{\sum_{i=1}^{\mathrm{n}}\left(x_{i}-\bar{x}\right)^{2}}{n-1}}$ Por lo tanto, la desviación estándar de las disposiciones a pagar estimadas por cada individuo, en el marco de una encuesta de valoración contingente, se obtendría de manera directa, aplicando la siguiente ecuación:

$$
\widehat{s d}_{D A P}=\sqrt{\frac{\sum_{i=1}^{n}\left(\hat{C}_{i}-\widehat{D A P}\right)^{2}}{n-1}}
$$

Donde $\hat{C}_{i}$ es la disposición a pagar estimada por individuo, $\widehat{D A P}$ es la estimación de la media de la disposición a pagar (o denominado también como $E\left[C_{i}\right]$ ) y $n$ es el número de encuestados.

Comparando las expresiones mostradas en las ecuaciones (8) y (9) se puede apreciar las diferencias entre el error estándar y la desviación estándar de la DAP. Al respecto, la desviación estándar $\widehat{s d}_{D A P}$ mide la dispersión que se presenta en las estimaciones de la DAP obtenidas por cada individuo encuestado de la muestra original, mientras que el error estándar $s e^{*}{ }_{D A P}$ calcula la variabilidad del estimador de la DAP promedio, considerando las replicaciones bootstrap, bajo la conceptualización de muestreo repetido.

La desviación estándar $\widehat{s d}_{D A P}$ permitiría conocer el nivel de variabilidad que presentan las preferencias hacia un bien o servicio, medido a través de la DAP, de una determinada muestra de estudio. En cambio, el error estándar puede ser considerado como un indicador de robustez, debido a que mide la dispersión o variabilidad de la media estimada de la DAP. 


\section{Estimación y resultados}

Utilizando la información de las encuestas realizadas en MINAM (2013), en el presente trabajo se aplicó el método bootstrap para estimar los coeficientes del modelo LOGIT, permitiendo así calcular el valor de la DAP promedio y su correspondiente error estándar. Las estimaciones realizadas fueron programadas en el software R (versión 3.2.2) asumiendo un número de replicaciones bootstrap de 10000 (para mayor detalle ver Anexo).

Tabla 1. Estimación del Modelo LOGIT, utilizando datos de MINAM (2013)

\begin{tabular}{lcc}
\hline \multicolumn{1}{c}{ Variables } & $\begin{array}{c}\text { Modelo logit } \\
\text { original }\end{array}$ & $\begin{array}{c}\text { Modelo logit } \\
\text { con bootstrap }\end{array}$ \\
\hline MONTO & $\mathbf{- 0 , 5 8 2 5 * * *}$ & $\mathbf{- 0 , 6 1 5 7 * * *}$ \\
& $(0,0838)$ & $(0,0935)$ \\
Edad & $\mathbf{- 0 , 0 2 4 3 * *}$ & $\mathbf{- 0 , 0 2 5 5 * *}$ \\
& $(0,0087)$ & $(0,0097)$ \\
EDU (años) & $\mathbf{0 , 0 6 9 9 *}$ & $\mathbf{0 , 0 7 4 0 *}$ \\
& $(0,0297)$ & $(0,0352)$ \\
Suminist & $\mathbf{0 , 5 8 8 5 *}$ & $\mathbf{0 , 6 3 5 7 *}$ \\
& $(0,2528)$ & $(0,2734)$ \\
(continúa...) & $($ continúa...) & $($ continúa...) \\
Constante & $\mathbf{2 , 8 1 2 4 * * *}$ & $\mathbf{2 , 9 1 8 0 * *}$ \\
& $(0,8223)$ & $(0,9418)$ \\
\hline Número de & 470 & 470 \\
observaciones & - & 10000 \\
Muestras bootstrap & 5,0460 & 5,1039 \\
DAP promedio & $(1,2259)$ & - \\
Desviación estándar & - & $(0,3914)$ \\
Error estándar & 0,1629 & 0,1940 \\
Pseudo R2 McFadden & Significancia estadistica: ‘***’0,001‘**0,01‘*’0,05 \\
Desviación estándar o error estándar en paréntesis ( )
\end{tabular}

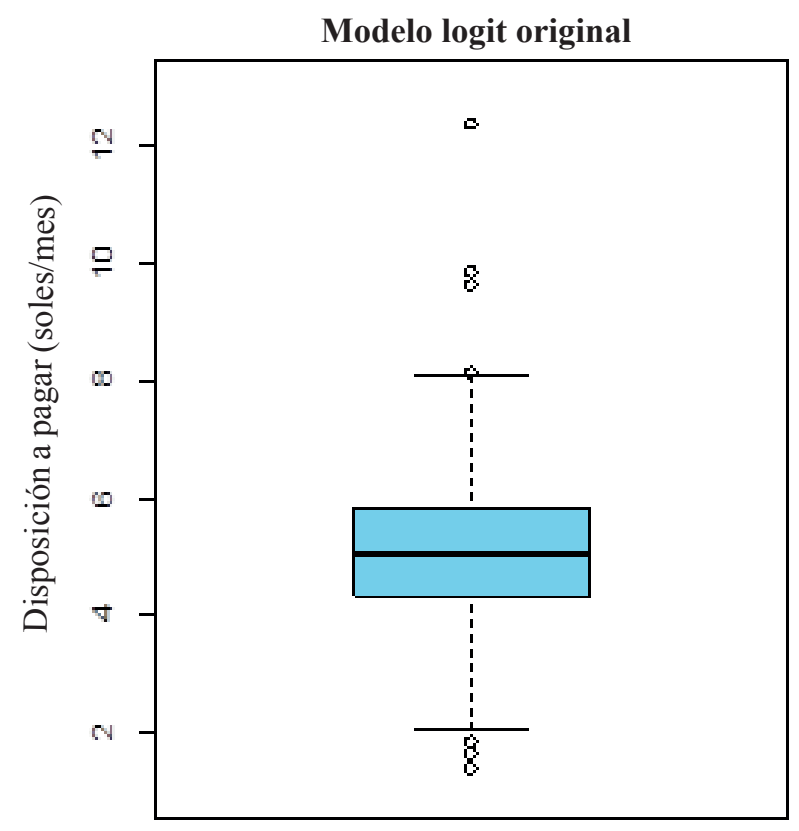

470 valores estimados
Es preciso señalar que en el estudio original no se evaluó la robustez de la DAP promedio calculada, debido a que no se estimó un indicador de dispersión o variabilidad del estimador. No obstante, al aplicarse el método bootstrap en las estimaciones del modelo LOGIT, fue posible calcular los errores estándar de la DAP, mediante la utilización de la ecuación (8).

Al respecto, como se observa en la Tabla 1, se pudo replicar el valor de la DAP promedio a 5,05 soles mensuales como en el estudio original, con una desviación estándar de 1,23 . Por otro lado, la DAP promedio estimada mediante bootstrap alcanzó los 5,10 soles mensuales, con un error estándar de 0,39. Es preciso destacar que la desviación estándar y el error estándar son medidas de dispersión que presentan distintas interpretaciones, por lo que sus valores distan notablemente uno de otro.

La desviación estándar obtenida en el primero modelo, da a conocer la dispersión promedio de las DAP estimadas por cada uno de los 470 encuestados. Por otro lado, el error estándar obtenido mediante el segundo modelo, se interpreta como la variabilidad que presenta la DAP promedio estimada dentro de las 10000 réplicas bootstrap.

La razón de que la desviación estándar obtenida resulte mayor al error estándar estimado, es debido a que la variabilidad entre los individuos encuestados es más alta que la dispersión entre las 10000 réplicas bootstrap estimadas de la DAP promedio. Este hecho se puede apreciar en la Figura 2, donde el rango intercuartílico de la distribución de las DAP estimadas (lado izquierdo), es más alto que el observado en la distribución de las replicaciones bootstrap (lado derecho).

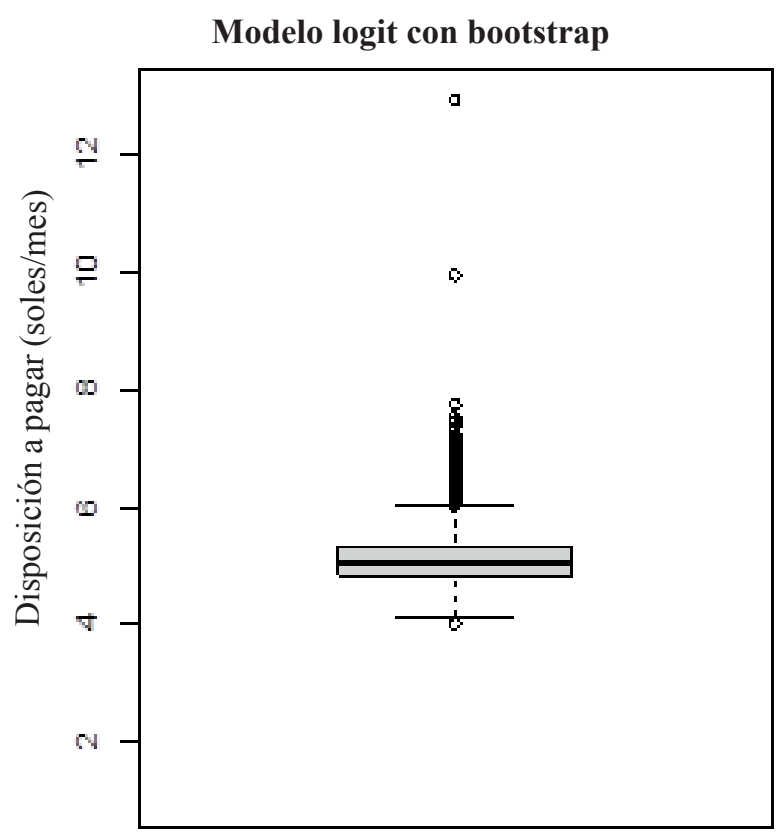

10000 replicaciones bootstrap

Figura 2. Distribución de la DAP estimada según modelo LOGIT utilizado 
Al tener los valores estimados de las replicaciones bootstrap, se puede obtener de manera directa el intervalo de confianza de la DAP. En la Tabla 2 se muestra las estimaciones realizadas de los intervalos de confianza bootstrap, mediante los métodos estándar ${ }^{11}$ $\mathrm{y}$ de percentiles ${ }^{12}$, tales como se muestran en Efron y Tibshirani (1993). Asimismo, con fines comparativos, se estimó también el intervalo de confianza aplicando el método desarrollado por Krinsky y Robb (1986) ${ }^{13}$.

Tabla 2. Estimación de los intervalos de confianza de la DAP (Soles/mes)

\begin{tabular}{lccc}
\hline & \multicolumn{2}{c}{$\begin{array}{c}\text { Intervalos de confianza } \\
\text { bootstrap }\end{array}$} & $\begin{array}{c}\text { Krinsky y Robb } \\
\text { (1986) }\end{array}$ \\
\cline { 2 - 4 } & $\begin{array}{c}\text { Método } \\
\text { Estándar }\end{array}$ & $\begin{array}{c}\text { Método de } \\
\text { Percentiles } \\
{[2,5 \% ; 97,5 \%]}\end{array}$ & $\begin{array}{c}\text { Kro } \\
\text { (1) }\end{array}$ \\
\hline $\begin{array}{l}\text { Límite } \\
\text { inferior }\end{array}$ & 4,34 & 4,50 & 4,52 \\
$\begin{array}{l}\text { Límite } \\
\text { superior }\end{array}$ & 5,87 & 5,96 & 5,87 \\
\hline
\end{tabular}

${ }^{1 /}$ El z-tabular se obtuvo considerando un nivel de significación $(\alpha)$ del $5 \%$.

${ }^{2 /}$ Estimación basada en 10000 replicaciones con 95\% de nivel de confianza.

\section{Conclusiones y recomendación}

Es común observar que en los estudios de valoración contingente se muestren las estadísticas descriptivas de las disposiciones a pagar (DAP) estimadas por individuo u observación, en donde la media es considerada como el valor referencial de estudio. Asimismo, se presenta la desviación estándar como un indicador de dispersión de la variable. No obstante, la desviación estándar no reflejaría la dispersión del valor económico obtenido, medido como la DAP promedio, debido a que no mide la dispersión que se presenta en la distribución empírica de la DAP promedio, como si lo hace el error estándar.

La aplicación del método bootstrap en el proceso de estimación de la DAP, en el marco de la valoración contingente, permite obtener fácilmente la distribución empírica de la DAP y, por lo tanto, la posibilidad de estimar su error estándar y/o intervalo de confianza (Ver McLeod y Bergland, 1989; Cooper, 1994 y Yoo, 2011) de manera inmediata y directa.

Utilizando la base de datos del estudio del MINAM (2013), se pudo estimar la desviación estándar y el error estándar de la DAP, alcanzando valores de 1,23 soles y 0,39 soles, respectivamente. Para obtener el error estándar de la DAP, se utilizó el método bootstrap, con 10000 réplicas aplicando la misma especificación funcional del

${ }^{11}$ Efron y Tibshirani (1993) lo denominan como confidence intervals based on bootstrap "table". Para calcular dicho intervalo de confianza se aplicó la siguiente fórmula: $I C(D A P)=\left[D A P^{*} \pm z_{1-2} s e^{*}\right]$.

${ }^{12} \mathrm{Si}$ definimos como la distribución empírica acumulada de los estimados DAP bootstrap, el intervalo de confianza tendrá la siguiente expresión: $\operatorname{IC}(D A P)=\left[F_{D A P}^{-1} *(\alpha / 2) ; \hat{F}^{-1}{ }_{D A P} *(1-\alpha / 2)\right]$.

${ }^{13} \mathrm{El}$ intervalo de confianza, basando en el método de Krinsky y Robb (1986), se estimó mediante el software STATA versión 13.1 utilizando el comando wtpcikr. estudio original. Por tanto, se recomienda no utilizar la desviación estándar como un indicador de dispersión para el valor económico estimado, debido a que estaríamos sobrevalorando el nivel de variabilidad.

\section{Literatura citada}

Bliemer, M. C. J., and Rose, J. M. 2013. Confidence intervals of willingness-to-pay for random coefficient logit models. Transportation Research, B 58, 199-214.

Bishop, R. C., and Heberlein, T. A. 1979. Measuring of extra market goods: are indirect measures biased? American Journal of Agricultural Economics, 61 (5), $1-15$.

Cameron, T. A. 1991. Interval estimates of non-market resource values from referendum contingent valuation surveys. Land Economics, 67 (4), 413-412.

Carson, R. T., and Czajkowski, M., 2013. A new baseline model for estimating willingness to pay from discrete choice models. International Choice Modelling Conference, Sydney.

Cooper, J. C. 1994. A comparison of approaches to calculating confidence interval for benefits measures from dichotomous choice contingent valuation surveys. Land Economics, 70 (1), 111-122.

Efron, B. 1979. Bootstrap methods: Another look at the jackknife. The Annals of Statistics, 7 (1), 1-26.

Efron, B., and Tibshirani, R. 1993. An introduction to the bootstrap. Chapman \& Hall/ CRC Press Company.

Gatta, V., Marcucci, E., and Scaccia, L., 2014. Willingness to pay confidence interval estimation methods: comparisons and extensions. CREI Working Paper $N^{\circ} 3 / 2014$

Hanemann, M. 1984. Welfare evaluation in contingent valuation experiments with discrete responses. American Journal of Agricultural Economics, 66 (3), 332-341.

Hole, A. R. 2007. A comparison of approaches to estimating confidence intervals for willingness to pay measures. Health Economics, 16 (8), 827-840.

Krinsky, I., Robb, A. 1986. On approximating the statistical properties of elasticities. Review of Economic and Statistics, 68 (4), 715-719.

Loyola, R. 2007. Valoración del servicio ambiental de provisión de agua con base en la Reserva Nacional Salinas y Agua Blanca - Cuenca del río Chili (RNSAB). Instituto Nacional de Recursos Naturales - INRENA.

Loyola, R., Soncco, C., 2007. Valoración económica del efecto en la salud por el cambio en la calidad del agua en zonas urbano marginales de Lima y Callao. Consorcio de Investigación Económica y Social (CIES).

Bergland, O. 1989. The use of bootstrapping in contingent valuation studies. Working Paper, Department of Agricultural Economics, Oregon State University, Corvallis. 
MINAM. 2013. Diseño e implementación de un esquema de retribución por servicios ecosistémicos hidrológicos en la cuenca del río Cañete. Ministerio del Ambiente. Editorial Supergráfica EIRL. Lima - Perú.

Vásquez, F., Cerda, A., Orrego, S. 2007. Valoración económica del ambiente. Thomson Learning. Buenos Aires.

Yoo, J. W., 2011. Advances in nonmarket valuation econometrics: spatial heterogeneity in hedonic pricing models and preference heterogeneity in stated preference models. (Ph.D. Thesis Dissertation). Penn State University. 
ANEXO: Códigos y funciones programadas en el software R

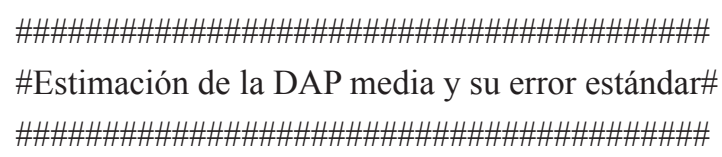

\# Modelo 1: Modelo logit \#

dap.R2=function(data) \{

col=length(data)

$\mathrm{n}=\operatorname{dim}($ data $)[1]$

$\operatorname{names}($ data $)[1]=" y "$

$\mathrm{y}=\operatorname{data}[[1]]$

model $=\operatorname{glm}(y \sim$., data $=$ data, family=binomial $(\operatorname{link}=" \operatorname{logit} "))$

betas $=$ model $\$$ coef

$\mathrm{m}=$ as.matrix (data)

for(i in $1: n) \mathrm{m}[\mathrm{i}, 1]=1$

$\mathrm{XB}=\mathrm{m} \% * \%$ betas

$\mathrm{e}=\exp (1)$

datar=rep $(1, n)$

datar $=$ data.frame(datar)

betar $<-\operatorname{glm}(y \sim$, data=datar, family=binomial $($ link="logit" $)) \$$ coef

$\log \mathrm{V} 1=0$

$\log \mathrm{V} 0=0$

for(i in $1: n)\{$

$\log \mathrm{V} 1=\log \mathrm{V} 1+\mathrm{y}[\mathrm{i}]^{*} \log \left(\mathrm{e}^{\wedge} \mathrm{XB}[\mathrm{i}] /\left(1+\mathrm{e}^{\wedge} \mathrm{XB}[\mathrm{i}]\right)\right)+(1-\mathrm{y}[\mathrm{i}]) * \log \left(1 /\left(1+\mathrm{e}^{\wedge} \mathrm{XB}[\mathrm{i}]\right)\right)$

$\left.\log \mathrm{V} 0=\log \mathrm{V} 0+\mathrm{y}[\mathrm{i}] * \log \left(\mathrm{e}^{\wedge} \operatorname{betar}[1] /\left(1+\mathrm{e}^{\wedge} \operatorname{betar}[1]\right)\right)+(1-\mathrm{y}[\mathrm{i}]) * \log \left(1 /\left(1+\mathrm{e}^{\wedge} \operatorname{betar}[1]\right)\right)\right\}$

$\mathrm{R} 2=\operatorname{as} . \operatorname{vector}(1-(\log \mathrm{V} 1 / \log \mathrm{V} 0))$

$\mathrm{m} \_2=\mathrm{m}[,-2]$

betas_2 $=$ betas[-2]

dap $=-\left(\mathrm{m} \_2 \% * \%\right.$ betas_2) $/$ betas[2]

dap.mean $=$ mean $($ dap)

return(list(DAP.mean=dap.mean, Coeff $=$ model $\$$ coef $)$ )

\}

\# Modelo 2: Modelo logit con bootstrap \#

boot.dap.R2=function(data,B, nivel=95) \{

$\operatorname{names}($ data $)[1]=" y "$

$\mathrm{y}=\operatorname{data}[[1]]$

alfa $=1-0.01 *$ nivel

$\mathrm{n}=\operatorname{dim}($ data $)[1]$

$\mathrm{p}=\operatorname{dim}($ data $)[2]$

dap.mean=dap.R2(data)\$DAP.mean

dapboot $=\operatorname{rep}(0, B)$

coefboot $=$ matrix $(0, B, p)$ 


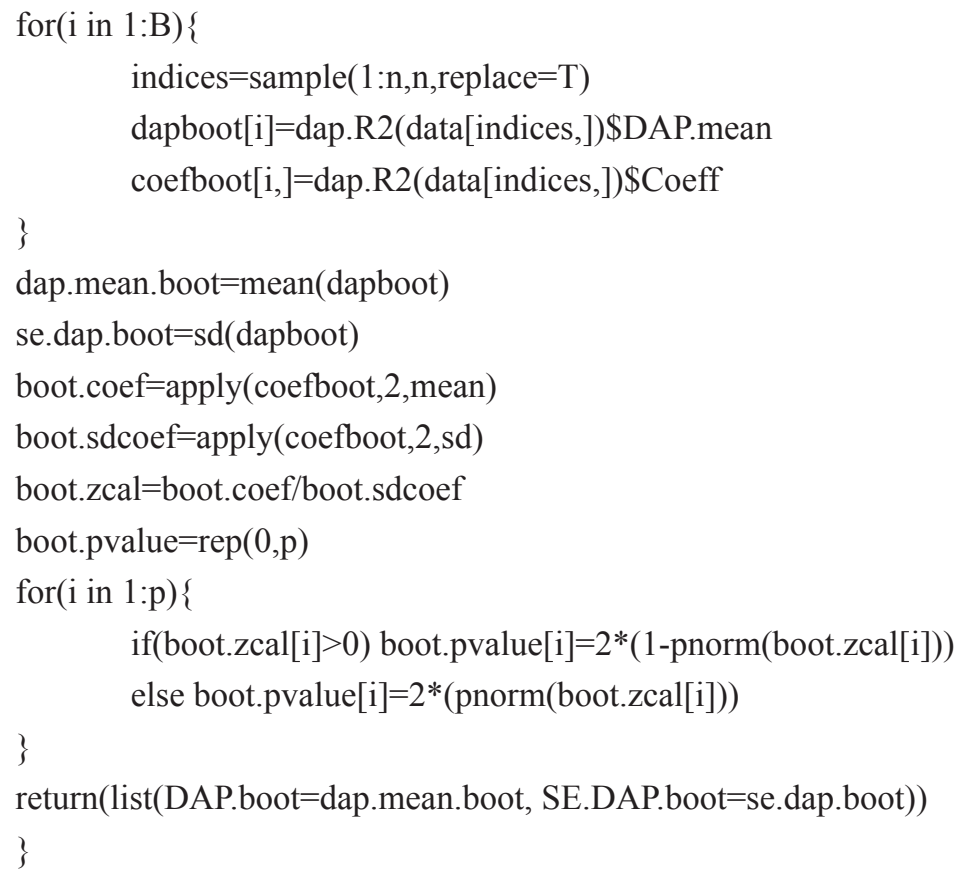

